\title{
Vias de acesso aos grandes vasos mediastinais no trauma torácico
}

\section{Surgical accesses to the major mediastinal vessels in thoracic trauma}

\author{
Roberto Gonçalves, TCBC-SP'; Roberto SAAD JÚnIOR, TCBC-SP²
}

RES U M O

\begin{abstract}
O traumatismo é a causa mais comum de mortes na população economicamente ativa e o trauma torácico é responsável direta ou indiretamente em um quarto destas mortes, aos quais as lesões de grandes vasos torácicos relacionam-se às mortes imediatas ou precoces no ambiente hospitalar. Dentro dos doentes que chegam vivos, pode-se dividi-los em estáveis ou instáveis, a via de acesso para a abordagem destes vasos irá depender desta situação, bem como de suas particularidades anatômicas, o que poderá implicar em incisões combinadas para uma adequada exposição. Neste artigo serão revisadas e discutidas as lesões destas estruturas e as vias acesso às mesmas.
\end{abstract}

Descritores: Ferimentos e lesões. Traumatismos torácicos. Aorta. Aorta torácica. Veia cava superior.

\section{INTRODUÇÃO}

A principal causa de morte em jovens com igual ou me nor idade de 40 anos é pelo trauma, sendo que o traumatismo de tórax está envolvido em aproximadamente $25 \%$ de todas estas mortes ${ }^{1,2}$. A epidemiologia da mortalidade por traumatismos, se dá através de três picos de uma curva trimodal, sendo o primeiro pico representado pelas mortes imediatas ao evento do trauma, o segundo pelas mortes precoces (até 3hs após o evento) e o terceiro pelas mortes tardias. As mortalidades relacionadas às lesões dos grandes vasos do tórax predominam nos dois primeiros picos.

Entende-se por grandes vasos torácicos, a aorta torácica, vasos braquiocefálicos, eixo das cavas torácicas, veias e artérias pulmonares e veia ázigos.

As lesões dos grandes vasos torácicos representam $10 \%$ de todas as lesões vasculares, $80 \%$ dos doentes que as possuem morrem no evento traumático e os sobreviventes que chegam ao hospital tem uma taxa de mortalidade acima de 20\%. Mais de $80 \%$ das lesões dos grandes vasos torácicos são causadas por trauma penetrante, e o sangramento resultante pode se apresentar de forma aguda exanguinante, contido por um hematoma e estruturas mediastinais, tamponamento cardíaco ou ainda na forma de fístula com estruturas adjacentes ${ }^{3}$.

\begin{abstract}
AVALIAÇÃO
$\mathrm{O}$ atendimento inicial deve seguir-se de modo hierarquizado segundo o protocolo do ATLS do comitê de trauma do colégio americano de cirurgiões. O mecanismo de trauma é de extrema relevância, assim sendo, os doentes que apresentarem trauma penetrante em região cérvicotorácica, precordial ou transfixante de mediastino, devem ser considerados como portadores de lesões mediastinais incluindo as vasculares até que se prove o contrário. Já no trauma contuso o mais importante a ser considerado é a energia envolvida no evento, sendo que as quedas de grandes alturas (acima de 10 metros) e as colisões de veículos automotores em alta velocidade (acima de $70 \mathrm{Km} / \mathrm{h}$ ) ainda são os mecanismos mais envolvidos, principalmente na ruptura traumática da aorta.

Doentes que apresentarem hipotensão arterial devem ser repostos com soluções cristalóides e transfusão sanguínea através de acessos venosos calibrosos em membros inferiores. Deve-se avaliar a necessidade de drenagem pleural, e na eventualidade de drenagem maciça (> $1500 \mathrm{ml}$ ) e persistência do choque apesar da reposição volumétrica, deve-se indicar a exploração cirúrgica de imediato. Tem-se descrito cada vez mais, que pacientes com lesão vascular penetrante e hipotensão branda podem beneficiar-se da "hipotensão permissiva", devendo-se evitar grandes reposições volêmicas antes do ato operatório, a
\end{abstract}

1. Mestre em Medicina pela Disciplina de Cirurgia Torácica da Faculdade de Ciências Médicas da Santa Casa de São Paulo FCMSCSP-SP-SP; 2. Professor Titular da Disciplina de Cirurgia Torácica do Departamento de Cirurgia da Faculdade de Ciências Médicas da Santa Casa de São Paulo FCMSCSP- SP. 
fim de se evitar um desprendimento de um coágulo perilesional desencadeando uma hemorragia fatal ${ }^{4,5}$.

\section{Propedêutica}

Achados clínicos que devem ser associados com lesão vascular torácica incluem: hipotensão arterial; tamponamento cardíaco com os componentes clássicos da tríade de Beck (estase jugular, hipotensão e hipofonese de bulhas); pseudocoarctação aórtica (pressão arterial desigual dos membros superiores em relação aos inferiores); desigualdade da pressão arterial entre os dois membros superiores; sopro ou frêmito interescapular; desvio traqueal por hematoma; estridor por compressão extrínseca de traquéia (lesão de carótida comum); hematomas supra claviculares; fratura de esterno e ou coluna torácica palpáveis; esmagamento torácico; Hemotórax volumoso.

Devemos salientar que o doente pode e muitas vezes não apresentar sintomas ou está oligosintomático ${ }^{6}$ devendo prevalecer a suspeita diagnóstica ditada pelo mecanismo de trauma, exigindo assim estudos complementares.

\section{Radiografia torácica}

Já na radiografia de tórax, muitos são os sinais que podem estar associados a este tipo de lesão ${ }^{7,8,1}$, principalmente no trauma aórtico, os mais discutidos são: alargamento de mediastino $>8 \mathrm{~cm}$ (posição ortostática); "borramento" do contorno aórtico; obliteração do espaço aórtico-pulmonar; relação entre as larguras do mediastino e tórax > 0,25; rebaixamento do brônquio fonte esquerdo; desvio traqueal para direita; desvio da sonda naso/oro gástrica para direita; hematoma extra-pleural apical ("boné apical)"; alargamento das linhas para vertebrais; alargamento da faixa paratraqueal; fratura do primeiro e segundo arcos costais (trauma de alta energia); fratura da escápula (trauma de alta energia); fratura de coluna torácica; fratura de esterno; hemotórax à esquerda; corpos estranhos ou suas trajetórias na projeção dos grandes vasos.

Vale a pena realçar que em uma pequena percentagem dos doentes portadores de trauma contuso de aorta e ramos supra aórticos, a radiografia de tórax pode estar sem alterações $(7 \%)^{9}$, sendo que mais uma vez a cinemática do trauma deverá orientar uma triagem diagnóstica adicional ${ }^{10}$.

\section{Tomografia Torácica X Arteriografia}

Até os anos noventa, a angiografia de subtração digital era considerada o padrão para o diagnóstico das lesões dos grandes vasos mediastinais, pois muitas vezes além do diagnóstico orientam o tratamento, seja por via convencional ou por técnica endovascular concomitante. Porém com os desenvolvimentos da tomografia computadorizada helicoidal multidetectores, tornaram o exame tomográfico mais rápido possibilitando reconstru- ções tridimensionais das estruturas em estudo com menor invasibilidade (angiotomografia), mas necessitando de profissional treinado para adequada interpretação do exame. ${ }^{11,12} \mathrm{Em}$ muitos serviços já define conduta sem outros exames, sendo a arteriografia reservada apenas para o procedimento endovascular, ${ }^{14,15}$.

\section{ABORDAGEM E TÁTICA OPERATÓRIA}

O sucesso no reparo cirúrgico vascular depende de um controle proximal e distal à lesão do vaso acometido. Em contraste ao abdome, em que a laparotomia mediana apresenta-se como via de acesso de eleição para correção de qualquer lesão abdominal, o tórax apresenta-se como um arcabouço semi-rígido osteocartilaginoso, recoberto por vários grupos musculares, no qual podem ser necessárias varias incisões para exposição dos diferentes vasos envolvidos no evento traumático e um equivoco na abordagem irá em acrescentar em perda de tempo, morbidade e mortalidade cirúrgicos.

Como discutido anteriormente, o doente pode apresentar-se em duas situações no momento da avaliação pré operatória: Instável ou estável do ponto de vista hemodinâmico.

\section{Doentes instáveis}

Após as manobras de reanimação iniciais, se a instabilidade for patente ou a lesão vascular for incerta, o doente deve ser rapidamente preparado para abordagem cirúrgica imediata.

As indicações de toracotomia na sala de emergência são:

Ferimento torácico penetrante principalmente em precórdio, com parada cárdio-respiratória testemunhada ou choque profundo à admissão, sendo os melhores resultados quando o agente vulnerante for uma arma branca e tratar-se de ferida cardíaca ${ }^{16}$. Os resultados da toracotomia na sala de emergência em traumatismos contusos são extremamente desalentadores, resultando dispêndio de recursos preciosos e exposição da equipe de emergência ${ }^{17}$.

Os objetivos da toracotomia de emergência ou reanimação são: evacuação de hemopericardio tamponante; controle temporário de hemorragia torácica exanguinante; compressões cardíacas internas; clampeamento aórtico para otimizar fluxo cerebral e coronariano; clampeamento do hilo pulmonar para prevenção de embolia aérea por grande laceração pulmonar.

A toracotomia deve ser inicialmente a anterolateral esquerda, na altura do $5^{\circ}$ espaço intercostal, desde a linha para esternal esquerda até o músculo grande dorsal na linha axilar posterior. A incisão deve seguir a borda superior da costela inferior evitando o feixe intercostal, o afastador de Finocheto deve ser colocado com a 
cremalheira voltada posteriormente a fim de deixar livre a parte anterior do tórax para eventual extensão da incisão (Figura 1).

A ampliação do campo operatório pode ser conseguida pela desarticulação de cartilagens condroesternais, e uma rápida pesquisa para avaliação de sangramento maciço no hemitorax direito pode ser realizada pela divulsão digital anteriormente ao coração através da pleura mediastinal. O cirurgião não deve protelar se houver sangramento que justifique abordagem contra lateral ou o campo operatório for restrito, devendo prolongar a incisão para uma bitoracotomia transesternal transversa (clamshell).

No momento da abertura torácica se for identificado hemopericardio tamponante, deve-se proceder rapidamente pericardiotomia paralela e anteriormente ao nervo frênico, com evacuação do saco pericárdico e na eventualidade de uma lesão ventricular pode-se prontamente oclui-la digitalmente ou com a introdução de uma sonda de Foley e insuflação de seu balão através da ferida. Em doentes que se apresentam em choque mesmo após evacuação pericárdica, pode-se realizar o clampeamento aórtico com pinças vasculares,que pode ser feito após a emergência da artéria subclávia esquerda ou na aorta justa diafragmática, sendo útil a manobra de introdução de sonda nasogástrica para distinguir o esôfago de uma aorta hipotensa.

As compressões cardíacas internas devem ser realizadas após a abertura do pericárdio, de maneira bimanual, comprimindo-se da base para o ápice cardíaco em movimentos semelhantes ao "bater palmas", ou comprimindo-se o coração contra a parede posterior do esterno, sendo condenada a compressão com uma só mão, pela possibilidade de perfuração miocárdica pelos dedos.

Em casos em que existam grandes lacerações pulmonares com sangramento ativo e grande fuga aérea, o controle pode ser obtido pelo pinçamento em massa do hilo pulmonar com um grande clampe vascular ${ }^{18}$, ou ainda se proceder à torção do pulmão sobre o eixo hilar em $180^{\circ}$ após a liberação digital do ligamento pulmonar ${ }^{19}$.

Grandes hematomas mediastinais situados no estreito superior torácico podem ser comprimidos por compressas para serem abordados posteriormente no centro cirúrgico ${ }^{20}$.

Deve-se enfatizar que estes procedimentos devem ser realizados no centro cirúrgico sempre que a fisiologia do doente permita, ou na impossibilidade deste fato, o doente deve permanecer o menor tempo possível na unidade emergência, sendo transferido o quanto antes para o bloco cirúrgico, local onde haverá recursos mais disponíveis para obtenção de melhores resultados.

Após a toracotomia, a hemostasia deve ser revista principalmente referente às artérias torácicas internas e vasos intercostais, o pericárdio deve permanecer aberto principalmente em casos de dilatação cardíaca, afim de se evitar uma restrição diastólica. Os hemitóraces devem ser drenados e o doente enviado a unidade de terapia intensiva.

\section{Doentes estáveis}

Estes doentes com suspeita de lesão vascular torácica devem ser submetidos ao estudo tomográfico (angiotomografia), podendo ser complementado pela arteriografia $^{21}$ na presença de lesão e dependendo da lo-

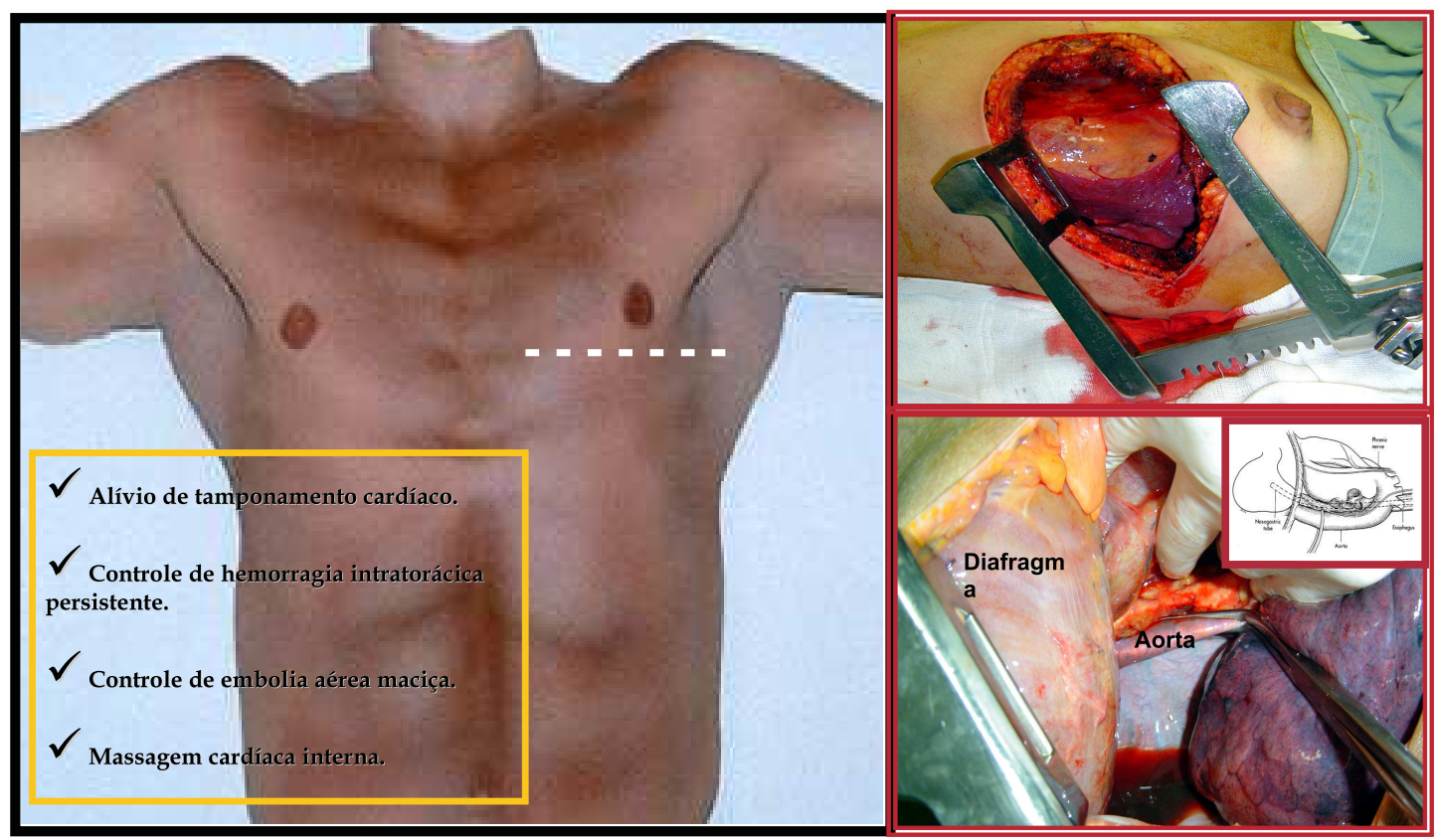

Figura 1 - Toracotomia anterolateral esquerda. Objetivos do procedimento. Nos detalhes, locação do afastador com cremalheira colocada posteriormente e clampeamento aórtico justa diafragmático. 
calização desta, diferentes incisões podem ser realizadas para adequada exposição e controle prévio do vaso com seu conseqüente reparo, como veremos a seguir.

\section{Aorta Ascendente e Croça aórtica}

Doentes portadores de lesões destes segmentos da aorta têm alta probabilidade de não chegarem vivos ao hospital. Dos sobreviventes a maioria possui trauma penetrante, e a taxa de sobrevivência dos doentes estáveis é de aproximadamente $50 \%{ }^{22}$. A via de acesso se dá pela esternotomia mediana, a qual pode ser estendida para as regiões cervicais para exposição dos ramos braquiocefálicos.A ligadura tática da veia braquiocefálica esquerda pode ser necessária para melhor apresentação, e na maioria das vezes um clampeamento lateral seguido de aortorrafia representa o tratamento, porém em lesões em que atinjam a parede posterior da aorta, podem exigir circulação extra-corpórea para sua correção.

\section{Aorta Descendente}

Normalmente os traumatismos da aorta descendente são acompanhados de lesões associadas em outros órgãos, trata-se do maior vaso torácico envolvido no trauma geral, a mortalidade pré hospitalar nas contusões é de $85 \%$ e dos sobreviventes não tratados que chegam ao hospital, a mortalidade pode ser de $1 \%$ por hora após 48 horas do evento traumático, ${ }^{23}$ sendo que $50 \%$ destes não apresentam sinais externos de trauma torácico ${ }^{24,25}$. O local mais comum da lesão no trauma contuso é no chamado istmo da aorta, após a emergência da artéria subclávia esquerda junto o ligamento arterioso, a lesão justa diafragmática e na raiz da aorta são locais menos comuns de lesão ${ }^{26}$. O trauma penetrante neste segmento, ocorre em $21 \%$ de todas feridas dos vasos torácicos, podendo ocorrer em qualquer ponto ao longo desta parte da aorta e sendo comum lesões associadas em outros órgãos, como esôfago brônquios e coração ${ }^{27}$.

A via de acesso é feita através de uma toracotomia póstero-lateral pelo $4^{\circ}$ espaço intercostal esquerdo, o controle proximal se faz ao redor do arco aórtico entre a artéria carótida e artéria subclávia esquerda e o distal, abaixo do hematoma perilesional tentando-se preservar o menor número de artérias intercostais para mobilização aórtica.

Vários métodos são descritos para o reparo, desde o bypass cardíaco esquerdo com bomba extra-corpórea, passando pelo shunt aórto-aórtico (shunt de Gott) até as técnicas sem shunt como "clampear e suturar". Dentro das modalidades de síntese pode-se proceder anastomose primária para lesões mais simples, interposição protética ou mais recentemente têm-se descrito cada vez mais a abordagem por radiologia intervencionista e técnica endovascular ${ }^{28,29}$,como discutiremos mais adiante. A principal complicação no pós-operatório é a paraplegia (aproximadamente $8 \%$ ) decorrente de vários fatores, entre os quais podemos citar: isquemia da medula espinhal por hipotensão e baixo fluxo no intra-operatório da artéria radicular magna (artéria de Adamkiewcz), situada entre $T_{9}$ e $\mathrm{L}_{1}$, edema medular e síndrome compartimental do canal raquiano. O risco maior se dá quando o tempo de clampeamento for maior que 30 minutos, hipotensão arterial e ligadura de vários segmentos de artérias intercostais $^{30}$ (Figura 2).

\section{Artéria e Veias Braquiocefálicas}

As lesões da artéria braquiocefálica representam o segundo grande vaso torácico mais acometido, ocorrendo em aproximadamente $9 \%$ no trauma penetrante e menos de $5 \%$ no contuso, neste a lesão geralmente situa-se na origem junto à aorta, já nas feridas a lesão pode ocorrer em qualquer extensão do vaso. A veia braquiocefálica esquerda está envolvida três vezes mais que a direita, devido seu maior tamanho e sua posição cruzando o mediastino superior ${ }^{31,2}$. Quanto a sintomatologia, os doentes podem apresentar déficits neurológicos, hipotensão severa ou mais raramente estar assintomáticos.

A via de acesso para abordagem destes vasos é através de uma esternotomia mediana com prolongamento para uma cervicotomia anterior direita (principalmente para lesão arterial). As lesões venosas podem ser reparadas ou ainda ligadas sem grande prejuízo para o doente, aliás, mais uma vez a ligadura tática da veia braquiocefálica esquerda amplifica a exposição para correção de uma lesão arterial subjacente.

O reparo arterial preferencial deve ser realizado por técnica de bypass aorto-inominada com prótese, sendo a anastomose proximal abaixo do hematoma (aorta ascendente), realizando-se em seguida a anastomose distal e por fim efetuando-se abertura do hematoma com fechamento da laceração. Em lesões com menor complexidade, pode-se optar por arteriorrafia lateral, porém avaliando-se o grau de estenose resultante sendo que as taxas de mortalidade nos doentes submetidos à cirurgia, podem chegar a 10\% e na eventualidade de ligadura arterial o índice de injuria neurológica é em torno de $25 \%{ }^{31}$.

A semelhança do trauma da aorta descendente, os stents e as endopróteses vasculares também tem tido sucesso no tratamento das lesões arteriais braquiocefálicas ${ }^{32}$. (Figura 3).

\section{Artérias e Veias subclávias}

O mecanismo de trauma predominante nestes vasos é o penetrante, representando o grande vaso torácico mais acometido neste tipo de traumatismo, 21\% nas artérias subclávias e $13 \%$ nas veias subclávias.

Um grande estudo 33 mostrou que a taxa de mortalidade nas lesões venosas foi significativamente maior que as lesões arteriais, $82 \%$ e $61 \%$ respectivamente, com uma taxa de mortalidade operatória global de 16\%.

No traumatismo torácico vascular contuso estes vasos podem estar lesados em $5 \%$ das vezes, e o mecanis- 

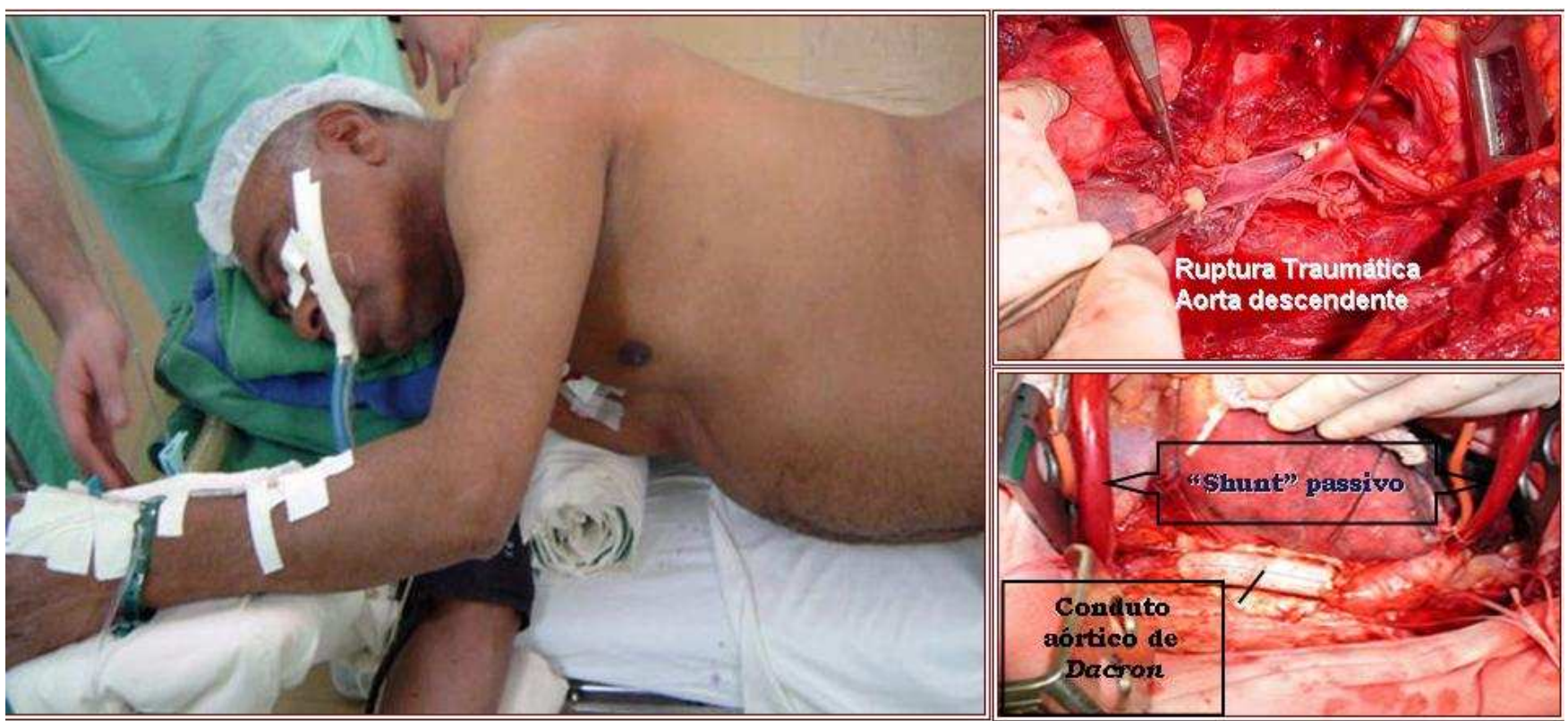

Figura 2 - Posicionamento do doente para realização de toracotomia póstero-lateral esquerda no $4^{\circ}$ espaço intercostal. Detalhes da aorta rota, locação de "shunt" passivo e reparo com conduto de dacron?.

mo de trauma mais comum é pelo cinto de segurança na colisão veículos automotores.

A apresentação clinica clássica de hematoma em expansão na região supra clavicular, sangramento profuso (no trauma penetrante), hemotórax, diferença de pulso entre os membros superiores com déficit neurológico, por lesão frequentemente associada do plexo braquial, nem sempre está presente, devendo o doente ser subme- tido a estudo angiográfico sempre que houver suspeita de injuria vascular.

A exposição para abordagem da lesão destes vasos é de extrema importância para o êxito. Devido a fragilidade destes vasos, estruturas nobres adjacentes e rica circulação colateral existente neste território, a dissecção deve ser cuidadosa e deve-se evitar as lesões iatrogênicas do plexo braquial e ner-

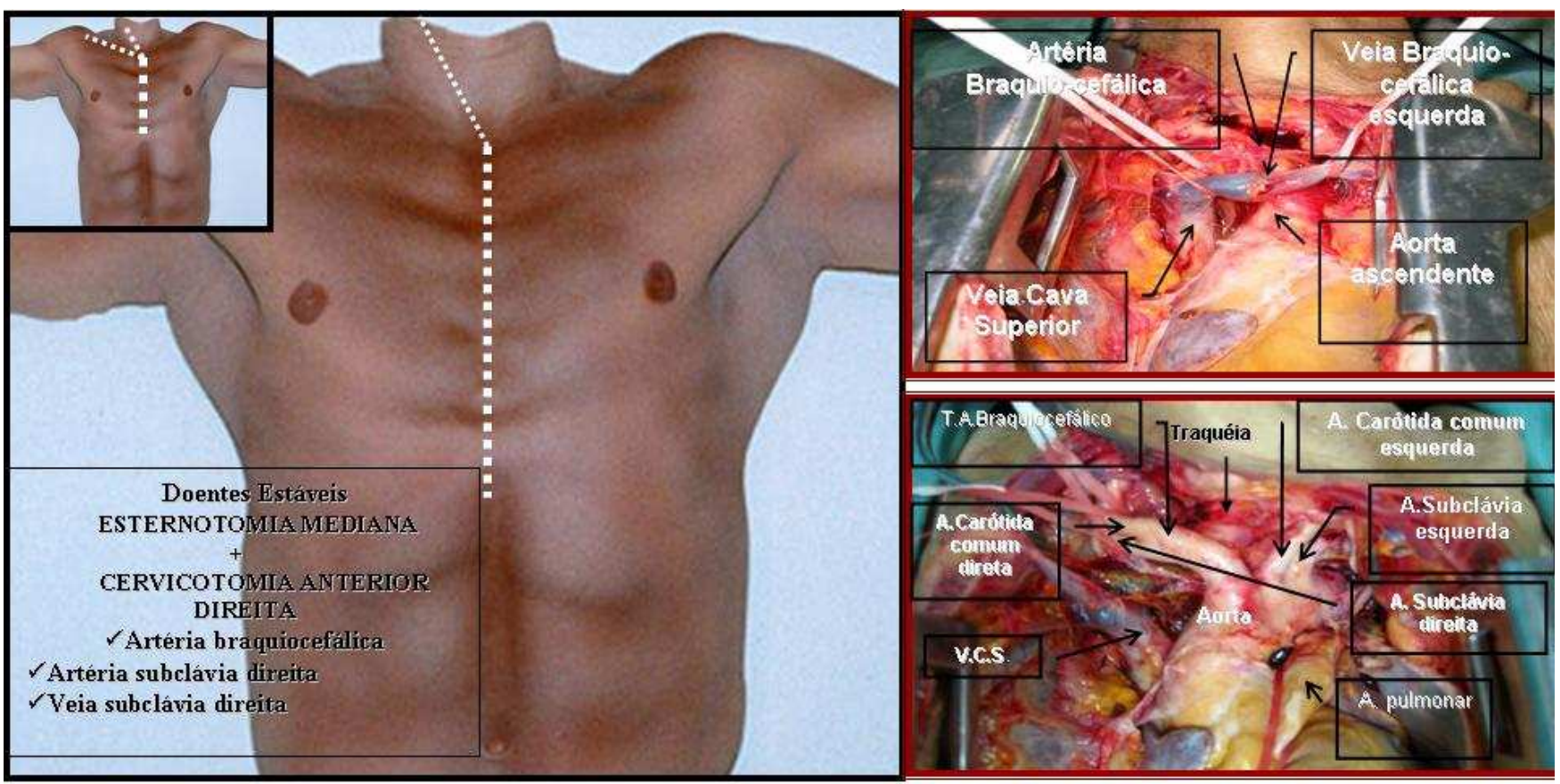

Figura 3 - Esternotomia com extensão cervical direita e vasos que esta via propicia abordar. Note cadarçamento, ligadura e rebatimento da veia braquiocefálica esquerda e sua relação com artéria braquiocefálica posterior. 
vos frênicos que estão em intimidade com estes vasos.

As lesões venosas na medida do possível devem ser reparadas, porém a ligadura pode ser necessária em casos extremos. Nas lesões arteriais pequenas, pode ser empregada a arteriorrafia lateral, mas nas mais complexas, a interposição com enxerto vascular será a regra, pois conseguir-se a anastomose término-terminal é conduta de exceção.

Para as lesões dos vasos subclávios à direita, uma esternotomia com extensão cervical anterior direita e ou uma incisão supra clavicular direita proporciona a melhor via de acesso para estes vasos. Já para as lesões subclavianas a esquerda, uma toracotomia ântero lateral alta no $3^{\circ}$ espaço intercostal esquerdo, permite o rápido controle proximal, mesmo digital, comprimindo o vaso contra a parte posterior da clavícula com posterior controle instrumental no trajeto intratorácico do vaso, no ápice pleural.Para o controle distal realiza-se incisão supra clavicular, podendose retirar a clavícula com algum grau de morbidade pós operatória.Em doentes mais estáveis, uma combinação de incisões supra e infraclaviculares evita a ressecção desta para o controle distal da lesão.A união da toracotomia com a incisão supra clavicular por uma esternotomia parcial, transforma o conjunto das incisões numa "toracotomia em livro aberto", por vezes necessária, porém com pouca amplificação do campo operatório, estiramento do plexo braquial e índice elevado de morbidade pós operatória com dor severa de difícil controle, razão pela qual deva ser evitada (Figura 4).

\section{Artéria Carótida Comum Esquerda}

As lesões neste vaso são relativamente raras, no trauma penetrante frequentemente apresenta lesões associadas em órgãos adjacentes (traquéia e esôfago), doentes com suspeita de lesão carotídea necessitam prontamente de via aérea definitiva, devido ao hematoma rapidamente expansivo com conseqüente obstrução da via aérea.

O prognóstico das lesões depende do estado neurológico do doente no momento da admissão e a taxa média de mortalidade é em torno de $17 \%$.

A abordagem operatória às lesões da carótida comum esquerda se faz pela esternotomia mediana e com extensão cervical anterior esquerda caso seja necessário e as táticas de reparo são semelhantes à abordagem da artéria braquiocefálica ${ }^{34}$ (Figura 5).

\section{Artérias e Veias Pulmonares}

Doentes portadores de lesões destes vasos têm uma alta de letalidade, algo superior a 70\%. O tipo de lesão mais comum é pelo trauma penetrante, sendo que a artéria pulmonar é lesada em $16 \%$ enquanto que as veias pulmonares são acometidas em $9 \%$ dos casos que chegam vivos ao pronto-socorro com trauma vascular torácico penetrante, e a semelhança com o traumatismo da aorta a maioria dos doentes com lesão destes vasos, morre no evento traumático.

A apresentação clinica mais comum é por hipotensão ou choque associado a hemotórax volumoso e

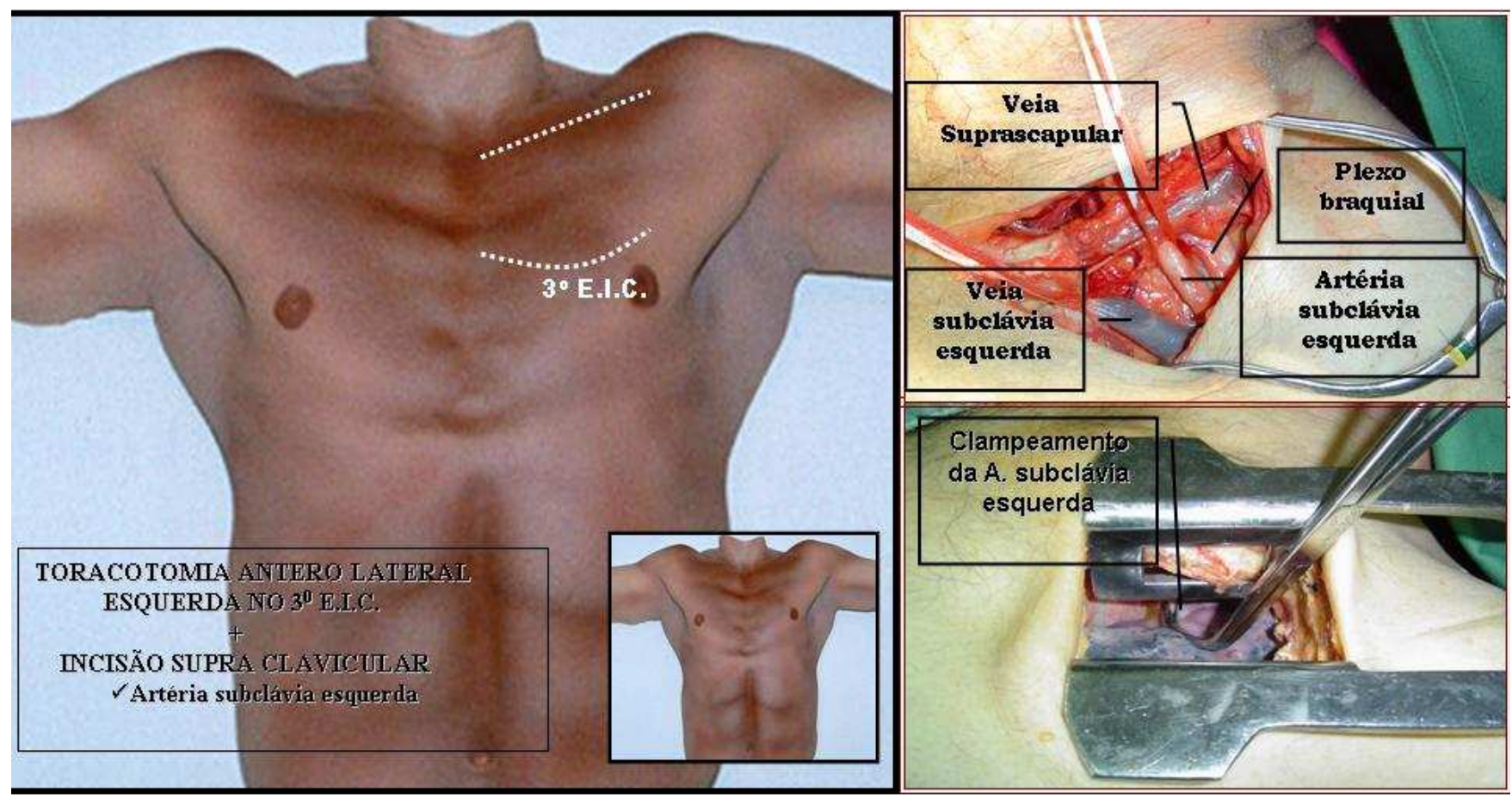

Figura 4 - Toracotomia anterior com incisão auxiliar supraclavicular para acesso à artéria subclávia esquerda.Em casos selecionados, as duas incisões podem ser unidas por uma esternotomia parcial. Nos detalhes pode-se observar as estruturas abordadas pelas duas incisões. 
alguns casos com hemoptise. No trauma contuso a hemorragia de uma artéria pulmonar, pode apresentar-se por alargamento mediastinal, tamponamento cardíaco, se a lesão estiver intra-pericárdica, ou hemotórax que se estabiliza após drenagem pleural mais que se reinicia profusamente após a reposição volêmica, minutos ou horas após a drenagem. Tal fato explica-se pelo sangramento de baixa pressão deste vaso e ainda em um doente em choque, que com a reexpansão da volemia com conseqüente aumento da pré-carga e pressão arterial,o vaso que por ventura estaria tamponado voltaria sangrar após a pressão deste suplantar a pressão intratorácica.

A via de acesso para as lesões das veias e segmentos distas das artérias pulmonares, são abordadas preferencialmente pela toracotomia póstero-lateral homolateral, já as lesões proximais da artéria pulmonar serão mais bem expostas pela esternotomia mediana. Um controle intra-pericárdico dos vasos pode ser útil e necessário, enquanto que as veias e artéria pulmonar esquerda sejam facilmente alcançadas após a abertura do saco pericárdico, a artéria pulmonar direita, exige a dissecção entre a veia cava superior e aorta ascendente para seu reparo.

Enquanto que pequenas lesões arteriais e venosas pulmonares posam ser tratadas por sutura lateral, lesões que envolvam ligadura de uma das artérias pulmonares exigem uma pneumonectomia enquanto a ligadura de uma veia pulmonar exige lobectomia correspondente $35,36,37$.

\section{Veia Cava Torácica e Veia ázigos}

As lesões da veia cava intratorácica possuem uma taxa de mortalidade acima de $60 \%$, tal índice elevado deve-se às lesões associadas, velocidade de fluxo sanguíneo, tamponamento cardíaco, hemotórax, hemoperitôneo maciços e dificuldade de exposição principalmente no que se refere à veia cava inferior, onde podem ser necessárias manobras que raramente podem levar ao sucesso tais como exclusão vascular total hepática, shunt atriocaval ou circulação extracorpórea com canulação pelos vasos femorais, com posterior reparo da lesão caval inferior através de uma atriotomia direita e reparo do vaso pela parte interna deste ${ }^{2,38}$. Uma esternotomia mediana dá acesso ao eixo das cavas, devendo-se proceder ao cadarçamento destas após a pericardiotomia. O clampeamento total da veia cava superior pode causar edema cerebral com aumento da pressão intracraniana, enquanto que a obstrução ao fluxo da veia cava inferior diminui significativamente a pré-carga, em que quando associado à hipotensão arterial pode desencadear uma parada cardíaca.

Como regra a venorrafia lateral pode ser empregada mesmo quando leva a certo grau de estenose na veia cava superior, porém em situações mais complexas devem-se empregar técnicas como bypass entre a veia braquiocefálica esquerda e cava superior proximal ou a interposição protética com Dacron ou politetrafluoroetileno expandido (PTFE) ${ }^{39}$.

As lesões da veia ázigos também possuem alta taxa de mortalidade (acima de 50\%), pelos mesmos moti-

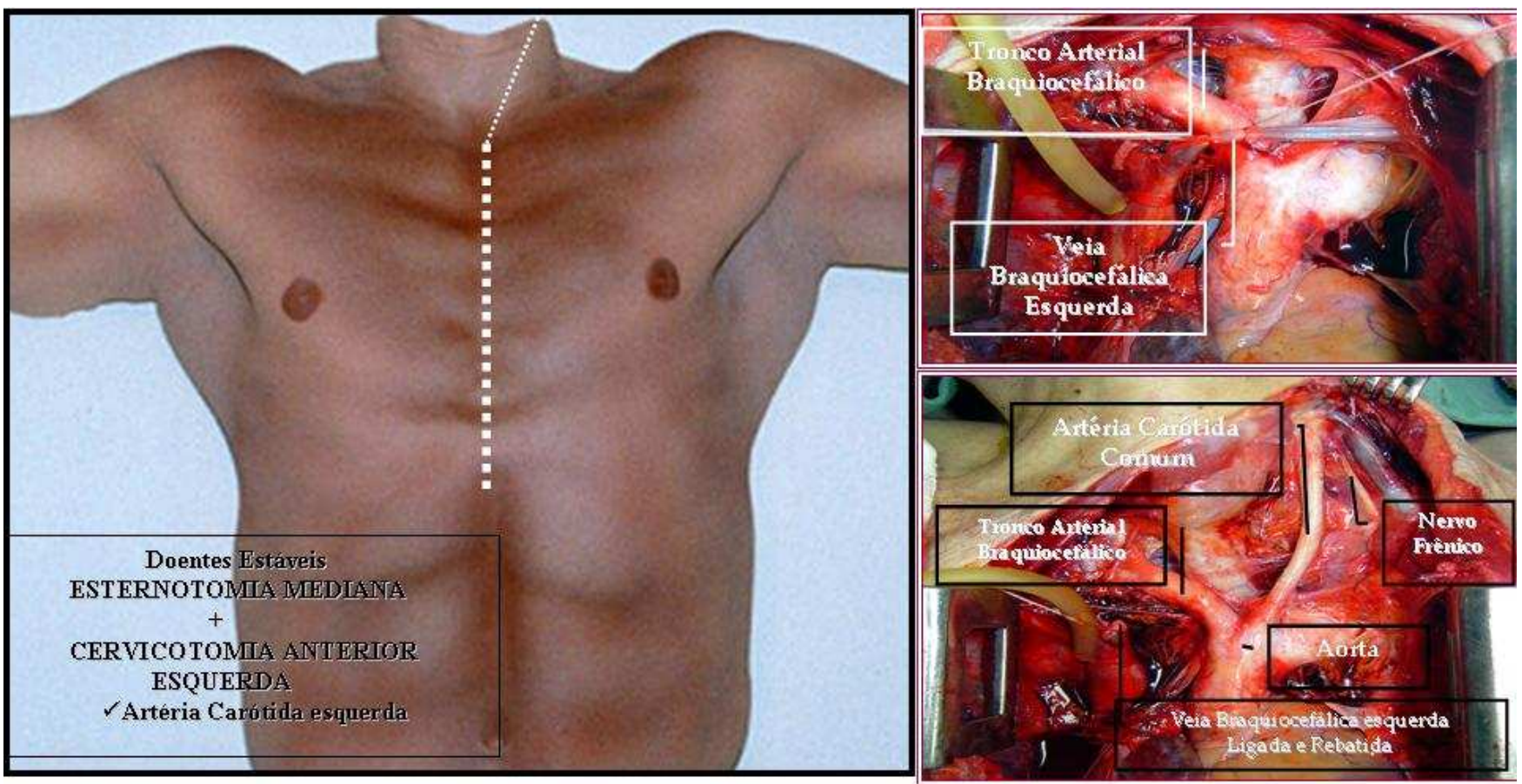

Figura 5 - Esternotomia mediana com extensão cervical esquerda dando amplo acesso a artéria carótida esquerda, após ligadura tática da veia braquicefálica esquerda. 


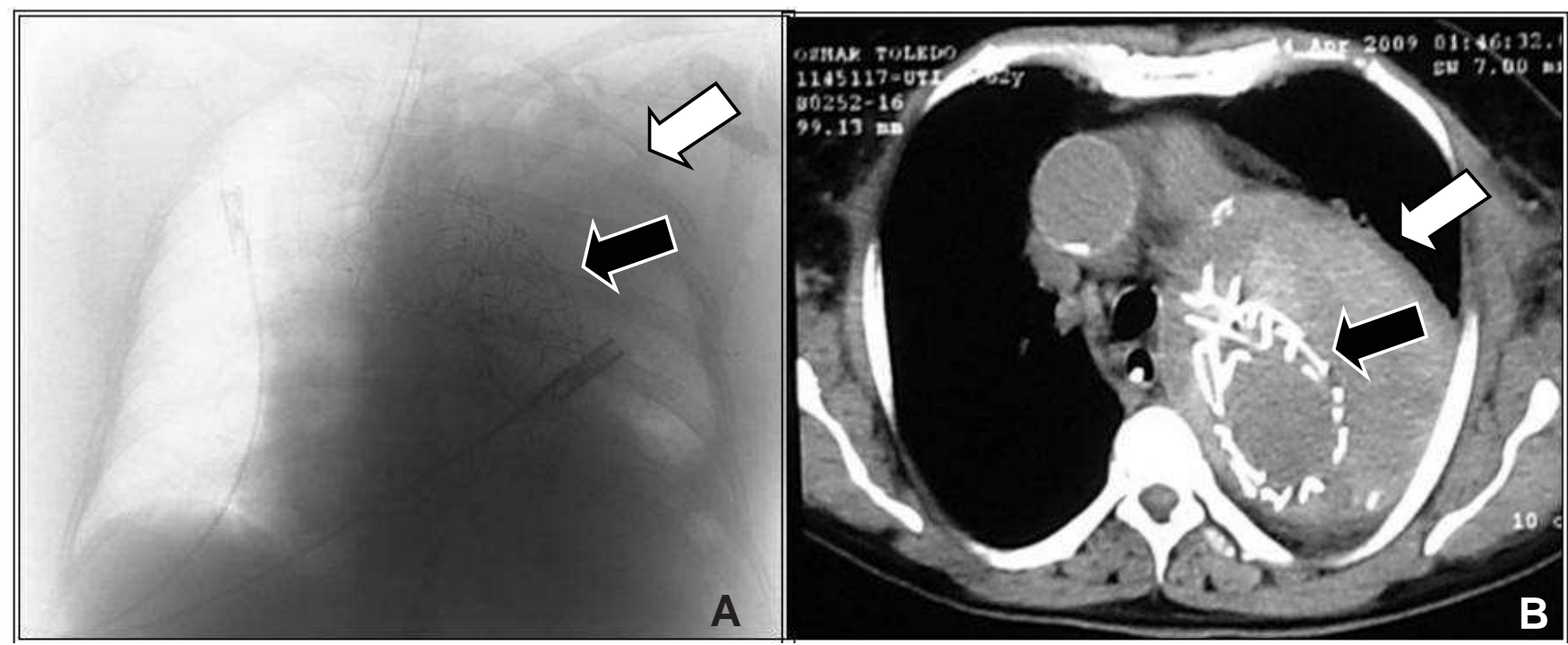

Figura 6 - Aspectos de ruptura traumática da aorta descendente, tratada por acesso endovascular. Note a endoprótese (seta negra) e hematoma perilesional (seta branca) observados tanto na radiografia (Figura 6A) como na tomografia computadorizada (Figura 6B).

vos descritos acima, tendo as lesões associadas como regra. Doentes portadores deste tipo de lesão devem sistematicamente ter os órgãos adjacentes explorados para exclusão de lesão despercebida, o que agravaria mais ainda o prognóstico do doente.

A via de acesso que melhor aborda este vaso é a toracotomia póstero-lateral direita e a identificação de lesão deste vaso autoriza sua ligadura dupla com conseqüente exposição da traquéia, carina e esôfago, órgãos comumente associados neste tipo de lesão ${ }^{40,41}$

\section{ABORDAGEM ENDOVASCULAR AOS VASOS TORÁCICOS}

A complexidade das vias de acesso convencional, associada à maior perda de sangue, dor e morbidade relativa, levou grupos a experimentar técnicas relativamente novas a este tipo de trauma. Injúrias associadas à lesão aórtica estão presentes em mais de $90 \%$ dos casos, sendo que aproximadamente um quarto destas lesões tem prioridade sobre o reparo aórtico. O manejo não operatório inicial pode ser indicado, em especial em centros onde existam protocolos para a não progressão da lesão da aorta com uso de beta- bloqueadores, vasodilatadores e disponibilidade de técnica endovascular, com menor trauma operatório após o estabelecimento da homeostase e das lesões prioritárias ${ }^{21,14,42,43}$.

A cirurgia endovascular, por se tratar de método minimamente invasivo e possibilidade do uso de anestesia local possui índices menores de reposição de sanguínea, paraplegia e mortalidade pós operatória, na cirurgia do trauma de aorta, quando comparada à técnica aberta $29,44,45$. Porém existem limitações ao uso desta técnica e dentro destas, talvez a mais importante, seja a necessidade de estabilidade hemodinâmica para que possa ser realizada. Outro ponto que merece ser discutido é o fato de a aorta do doente traumatizado, que tipicamente é um doente jovem, possui um calibre menor com ângulo (arco) aórtico mais agudo quando comparado a órgãos aneurismáticos, para os quais as endopróteses atuais foram projetadas, tornando menos ideal o uso deste dispositivo na cirurgia do trauma. Além disso, não se tem informações a respeito da durabilidade destas próteses, o que é desejável, pela sobrevida longa do paciente em questão ${ }^{45}$.

Dentre as complicações relacionadas ao uso de endoprótese aórtica no trauma podemos citar: "endoleaks" maiores quando comparados às aortas aneurismáticas com taxas conversão igualmente maiores; abaulamento da prótese, fratura, migração; oclusão inadvertida pela prótese da artéria carótida e subclávia esquerda; acidente vascular isquêmico por manipulação do cateter e paraplegia ${ }^{45,46}$ (Figura 6).

Vários trabalhos têm mostrado também a utilização da técnica endovascular, seja pura ou combinada com a técnica aberta, para abordagem dos troncos supraaórticos, em especial as artérias subclávias ${ }^{47}$. Entre os critérios de potencial indicação para sua utilização estão: falsos aneurismas, fístulas arteriovenosas, "flap" intimal e técnica híbrida com uso de balão de oclusão endovascular proximal à lesão ${ }^{48,49}$. Sendo que as atuais situações de contra-indicação do método podemos citar: hipotensão refratária á reposição de volume, síndrome compartimental do membro com compressão neuro-vascular, transecção total ou subtotal do vaso, pontos insuficientes de fixação proximal e distal do stent e segmento arterial traumatizado longo ${ }^{48,50}$.

Os resultados preliminares destes estudos mostram que o reparo endovascular pode ser usado numa fração de doentes com este tipo de lesão, e quando compa- 
rados com a técnica convencional, apresentam maior beneficio quanto à menor perda sanguínea; tempo reduzido do ato operatório; menor dissecção de estruturas anatômicas como plexo braquial, nervos frênico, laringeo recorrente, vago e veias braquiocefálicas com diminuição das taxas de lesões iatrogênicas ${ }^{49,50,51}$

Porém complicações relacionadas a este tipo de abordagem operatória ao traumatismo dos troncos braquiocefálicos são relatados em diversas séries ${ }^{47,49,50,51}$.Trombose, estenose, fratura e deslocamento do stent recoberto; oclusão inadvertida da artéria vertebral, bem como embolização cerebral e lesões concomitantes da artéria e veia subclávia por manipulação do fio guia estão entre as mais graves destas situações ${ }^{52}$.

As técnicas de abordagem endovascular para os traumatismos torácicos ainda necessitam maior experiência e de estudos prospectivos controlados para saber seu papel pleno. Porém já podemos observar seu crescente uso em situações cada vez mais complexas, sendo que seu conhecimento e indicações, aliadas ás técnicas convencionais, sejam um instrumento a mais para o cirurgião que se depara com doença tão complexa.

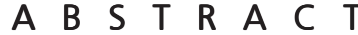

Trauma is the most common cause of death in the economically active population and thoracic trauma is directly or indirectly responsible for one quarter of these deaths. Lesions to the large thoracic vessels are associated with immediate or early death in the hospital setting. Patients admitted alive can be classified as stable or unstable. The access route to be elected for management of these veins will depend on this status, as well as on the anatomical particularities of the patient, which may require combined incisions for adequate access. This article provides a review and discussion of lesions to these structures as well as access routes to them.

Key words: Wounds and injuries. Thoracic injuries. Aorta. Aorta, thoracic. Vena cava, superior.

\section{REFERENCIAS}

1. ATLS - Advanced Trauma Life Support for Doctors. Student Course Manual. 8th ed. American College of Surgeons Committee on Trauma; 2008. Thoracic trauma; p. 85-110.

2. Coimbra RSM, Saad Jr R. Trauma dos grandes vasos torácicos. In: Saad Jr R, editor. Trauma de tórax. São Paulo: Robe; 1993. p.11941.

3. Mattox KL, Feliciano DV, Burch J, Beall AC Jr, Jordan GL Jr, De Bakey ME. Five thousand seven hundred sixty cardiovascular injuries in 4459 patients. Epidemiologic evolution 1958 to 1987. Ann Surg. 1989;209(6):628-705; discussion 706-7.

4. Bickell WH, Wall MJ Jr, Pepe PE, Martin RR, Ginger VF, Allen MK, et al. Immediate versus delayed fluid resuscitation for hypotensive patients with penetrating torso injuries. N Engl J Med. 1994:331(17):1105-9.

5. Mattox KL, Hirshberg A, Wall MJ Jr. Alternative approaches to resuscitation. In: Ivatury RR, Cayten CG, editors. The textbook of penetrating trauma. Baltimore, Md: Williams and Wilkins; 1996. p.195-205.

6. Mattox KL. Approaches to trauma involving the major vessels of thorax. Surg Clin North Am. 1989;69(1):77-91

7. Mirvis SE, Bidwell JK, Buddemeyer EU, Diaconis JN, Pais SO, Whitley $\mathrm{JE}$, et al. Value of chest radiograph in excluding traumatic aorta rupture. Radiology. 1987;163(2):487-93.

8. Woodring JH, King JG. Determination of normal transverse mediastinal width and mediastinal-width to chest-width $(\mathrm{M} / \mathrm{C})$ ratio control subjects: Implications for subjects with aortic brachiocephalic arterial injury. J trauma. 1989;29(9):1268-72.

9. Woodring $\mathrm{JH}$. The normal mediastinum in blunt traumatic rupture of the thoracic aorta and brachiocefalic arteries. J Emerg Med. 1990;8(4):467-76

10. Feliciano DV, Rozycki GS. Advances in the diagnosis and treatment of thoracic trauma. Surg Clin North Am. 1999;79(6)1417-29.

11. Ahrar K, Smith DC, Bansal RC, Razzouk A, Catalano RD. Angiography in blunt thoracic aortic injury. J Trauma. 1997;42(4):665-9.

12. Gavant ML, Menke PG, Fabian T, Flick PA, Graney MJ, Gold RE. Blunt traumatic aortic rupture: detection with helical $C T$ of the chest. Radiology. 1995;197(1):125-33.
13. Mirvis SE, Shanmuganathan K, Buell J, Rodriguez A. Use of spiral computed tomography for the assessment of blunt trauma patients with potential aortic injury. J Trauma. 45(5):922-30.

14. Gavant ML. Helical CT grading of traumatic aortic injuries. Impact on clinical guidelines for medical and surgical management. Radiol Clin North Am. 1999;37(3):553-74, vi.

15. Mirvis SE. Thoracic vascular injury. Radiol Clin North Am. 2006;44(2):181-97, vii.

16. Ivatury RR, Shah PM, Ito K, Ramirez-Schon G, Suarez F, Rohman $M$. Emergency room thoracotomy for the resuscitation of patients with "fatal" penetrating injuries of the heart. Ann Thorac Surg. 1981;32(4):377-85

17. Working Group, Ad Hoc Subcommittee on Outcomes, American College of Surgeons. Committee on Trauma. Practice management guidelines for emergency department thoracotomy. Working Group, Ad Hoc Subcommittee on Outcomes, American College of Surgeons-Committee on Trauma. J Am Coll Surg. 193(3):303-9.

18. Van Natta TL, Smith BR, Bricker SD, Putnam BA. Hilar control in penetrating chest trauma: a simplified approach to an underutilized maneuver. J Trauma. 2009;66(6):1564-9.

19. Wilson A, Wall MJ Jr, Maxson R, Mattox K. The pulmonary hilum twist as a thoracic damage control procedure. Am J Surg. 2003;186(1):49-52.

20. Wall MJ Jr, Hirshberg A, LeMaire SA, Holcomb J, Mattox K. Thoracic aortic and thoracic vascular injuries. Surg Clin North Am. 2001;81(6):1375-93.

21. Mattox KL, Walkes JC. Advances in the management of thoracic vascular injury. Scan J Surg. 2002;91:46-51.

22. Demetriades D. Penetrating injuries to the thoracic great vessels. J Card Surg. 1997;12(2 Suppl):173-9; discussion 179-80.

23. Parmley LF, Mattingly TW, Manion WC, Jahnke EJ Jr. Nonpenetrating traumatic injury of the aorta. Circulation. 1958;17(6):1086-101.

24. Merrill WH, Lee RB, Hammon JW Jr, Frist WH, Stewart JR, Bender HW Jr. Surgical treatment of acute traumatic tear of the thoracic aorta. Ann Surg.1988;207(6):699-706.

25. Schepens MA, Van Cauwelaert PA, Gerad YE. Acute traumatic disruption of the aorta. Acta Chir Belg. 1989;89(4):189-95 
26. Reardon MJ, Hedrick TD, Letsou GV, Safi HJ, Espada R, Baldwin JC. CT reconstruction of an unusual chronic posttraumatic aneurysm of the thoracic aorta. Ann Thorac Surg. 1997;64(5):14802.

27. Feliciano DV. Trauma to the aorta and major vessels. Chest Surg Clin N Am. 1997;7(2):305-23.

28. Nzewi O, Slight RD, Zamvar V. Management of blunt thoracic aortic injury. Eur J Vasc Endovasc Surg. 2006;31(1):18-27.

29. Demetriades D, Velmahos GC, Scalea TM, Jurkovich GJ, KarmyJones $R$, Teixeira PG, et al. Diagnosis and treatment of blunt thoracic aortic injuries: changing perspectives. J Trauma. 2008;64(6):14158; discussion 1418-9

30. von Oppel UO, Dunne TT, De Groot MK, Zilla P. Traumatic aortic rupture: twenty-year metaanalysis of mortality and risk of paraplegia. Ann Thorac Surg. 1994;58(2):585-93.

31. LeMaire SA, Conklin DL, Wall MJ Jr. Penetrating thoracic vascular injuries. In: Rich NM. Mattox KL, Hirshberg A, editors. Vascular trauma. Cidade: Elsevier; 2004. p.251-67.

32. du Toit DF, Strauss DC, Blaszczyk M, de Villiers R, Warren BL. Endovascular treatment of penetrating thoracic outlet arterial injuries. Eur J Endovasc Surg. 2000;19(5):489-95.

33. Demetriades $D$. Penetrating injuries to the thoracic great vessels J Card Surg. 1997;12(2 Suppl):173-9; discussion 179-80

34. Asensio JA, Valenziano CP, Falcone RE, Grosh JD. Management of penetrating neck injuries. The controveversy surrounding zone ॥ injuries. Surg Clin North Am. 1991;71(2):267-96.

35. Ambrose G, Barrett LO, Angus GL, Absi T, Shaftan GW. Main pulmonary artery laceration after blunt trauma: accurate preoperative diagnosis. Ann Thorac Surg. 2000;70(3):955-7.

36. Rai VK, Malireddy K, Dearmond D, Myers J, Dent DL. Traumatic pseudoaneurysm of the pulmonary artery. J Trauma. 2010;69(3):730.

37. Quartey B, Jessie E. Pulmonary artery and vein pseudoanerysm after gunshot to the chest. J Emerg Trauma Shock. 2011;4(2):3136.

38. Couves $C M$, Heughan C. Laceration of superior vena cava due to blunt trauma: successful surgical management. Can T Surg. 1981;24(4):402-3.

39. Bakaeen FG, Wall MJ Jr, Mattox KL. Successful repair of an avulsion of the superior vena cava from the right atrium inflicted by blunt trauma. J Trauma. 2005;59(6):1486-8.

40. Walz M, Kolbow B, Auerbach F, Fernandez-Laser C, Eppen R Rupture of the azygos vein by blunt thoracic trauma. A case report and literature review. Unfallchirurg. 2003;106(9):764-7.

41. Wall MJ Jr, Mattox KL, Debakey ME. Injuries to the azygos venous system. J Trauma. 2006;60(2):357-62
42. Wall MJ Jr, Granchi T, Liscum K, Mattox KL. Penetrating thoracic vascular injuries. Surg Clin North Am. 1996;76(4):749-61.

43. O'Connor JV, Byrne C, Scalea TM, Griffith BP, Neschis DG. Vascular injuries after blunt chest trauma: diagnosis and management. Scand J Trauma Resusc Emerg Med. 2009;17:42.

44. Amabile P, Collart F, Gariboldi V, Rollet G, Bartoli JM, Piquet P. Surgical versus endovascular treatment of traumatic thoracic aortic rupture. J Vasc Surg. 2004;40(5):873-9.

45. Xenos ES, Abedi NN, Davenport DL, Minion DJ, Hamdallah O, Sorial EE, et al. Meta-analysis of endovascular vs open repair for traumatic descending thoracic aortic rupture. J Vasc Surg. 2008;48(5):1343-51.

46. Rheaume P, Chen J, Casey P. Open vs endovascular repair of blunt traumatic thoracic aortic injuries. J Vasc Surg. 2010;51(3):763-9.

47. du Toit DF, Odendaal W, Lambrechts A, Warren BL. Surgical and endovascular management of penetrating innominate artery injuries. Eur J Vasc Endovasc Surg. 2008;36(1):56-62.

48. Danetz JS, Cassano AD, Stoner MC, Ivatury RR, Levy MM. Feasibility of endovascular repair in penetrating axillosubclavian injuries: a retrospective review. J Vasc Surg. 2005;41(2):246-54.

49. Xenos ES, Freeman M, Stevens S, Cassada D, Pacanowski J, Goldman M. Covered stents for injuries of subclavian and axillary arteries. J Vasc Surg. 2003;38(3):451-4.

50. Carrick MM, Morrison CA, Pham HQ, Norman MA, Marvin B, Lee $J$, et al. Modern management of traumatic subclavian artery injuries: a single institution's experience in the evolution of endovascular repair. Am J Surg. 2010;199(1):28-34.

51. Aksoy M, Tunca F, Yanar H, Guloglu R, Ertkin C, Kurtoglu M. Traumatic injuries to the subclavian and axillary arteries: a 13-year review. Surg Today. 2005;35(7):561-5.

Recebido em 10/10/2010

Aceito para publicação em 15/11/2010

Conflito de interesse: nenhum

Fonte de financiamento: nenhuma

\section{Como citar este artigo:}

Gonçalves R, Saad Júnior R. Vias de acesso aos grandes vasos mediastinais no trauma torácico. Rev Col Bras Cir. [periódico na Internet] 2012; 39(1). Disponível em URL: http://www.scielo.br/rcbc

\section{Endereço para correspondência:}

Roberto Gonçalves

E-mail: rgtorax@yahoo.com.br 